\title{
Hanseníase: o controle dos contatos no município de Londrina-PR em um período de dez anos
}

\author{
Leprosy: control of household contacts in the municipality of Londrina-PR for a ten-year period \\ Lepra: el control de los contactos en el municipio de Londrina-PR en un periodo de diez años
}

\section{Elma Matias Dessunti', Zeneide Soubhia', Elaine Alves', Cristina Maria Aranda', Maria Priscila Amed Ali Barro'}

'Universidade Estadual de Londrina. Departamento de Enfermagem. Londrina, PR

\author{
Submissão: 20/08/2008 \\ Aprovação: 10/10/2008
}

\section{RESUMO}

Estudo descritivo, com o objetivo de analisar variáveis relacionadas aos contatos de pacientes com hanseníase atendidos no município de Londrina, num período de dez anos. Os dados foram obtidos das fichas de comunicantes e do SINAN. Dentre os 1055 casos de hanseníase, foram registrados 3394 contatos, com média de 3,2. Os indivíduos mais expostos possuem até 40 anos de idade (71,5\%), sendo filhos/ a (40,6\%) e esposo/a (17,8\%). Foram examinados 173 I (51,0\%) contatos, dos Quais, 183 apresentavam algum sinal de hanseníase: confirmados 16 casos, descartados 47 e não concluíram a investigação $120(65,6 \%)$. A maioria dos contatos $(51,6 \%)$ foi exposta às formas multibacilares e 10, 1\% comprovaram a efetivação de duas doses da BCG. Conclui-se Que está havendo falhas no acompanhamento dos contatos.

Descritores: Hanseníase/epidemiologia; Vigilância epidemiológica; Período de transmissibilidade; Enfermagem.

\section{ABSTRACT}

The purpose of this descriptive study is to analyze variables related to leprosy patients' household contacts who received treatment in Londrina-PR-Brazil for a ten-year period. The data analysis was based on the health service's records and from a system of infectious disease. Out of 1055 leprosy's patients, it was recorded 3394 contacts with an average of 3,2. The most exposed individuals were those aged up to 40 (71,5\%); son/daughter (40.6\%) and husband/wife (17.8\%). Of the 173 I contacts (51.0\%) examined, 183 showed some signs of the disease: there were 16 confirmed cases, 47 were excluded and 120 did not finish the clinical investigation. Most of the contacts (51.6\%) were exposed to the multibacillary forms and $12.8 \%$ proved they were vaccinated with two doses of BCG. It is possible to conclude that the follow-up of the contacts was not adequate.

Descriptors: Leprosy/epidemiology; Epidemiologic surveillance; Communicable period; Nursing.

\section{RESUMEN}

Estudio descriptivo, con el objetivo de analizar variables relacionadas a los contactos de pacientes con Lepra Que son atendidos en el município de Londrina, en un período de diez años. Los datos fueron obtenidos en las fichas de los acompañantes y del SINAN (órgano del MInisterio de Salud Que registra la ocurrencia de toda enfermedad transmisible). Entre los 1055 casos de hanseníase, fueron registrados 3394 contactos, con una media de 3,2. Los individuos más expuestos tienen hasta 40 años de edad (71,5\%), siendo hijos/as (40,6\%) y esposos/as (17,8\%). Fueron examinados 173 I $(51$ 1,0\%) contactos, de los cuales, 183 presentaban alguna señal de la lepra: confirmados 16 casos, descartados 47 y sin concluir la investigación 120 . La mayoría de los contactos $(51,6 \%)$ fue expuesta a las formas multibacilares y 12,8\% comprobó la administración de dos dosis de la BCG. Se concluye Que está habiendo fallas en el acompañamiento de los contactos. Descriptores: Lepra/epidemiología; Vigilancia epidemiológica; Periodo de transmisión; Enfermería.

Correspondência: Elma Matias dessunti. Universidade Estadual de Londrina. Departamento de Enfermagem. Rodovia Celso Garcia Km 380. Campus Universitário. Cx. Postal 6001. CEP 86051-990. Londrina, PR. 


\section{INTRODUÇÃO}

A hanseníase, doença infecciosa e de período de incubação prolongado, tem, em seus contatos intradomiciliares, um importante meio para a manutenção da endemia. É considerada como relevante problema de saúde pública na maioria dos estados brasileiros, apesar dos esforços do Ministério da Saúde na tentativa de controlar a infecção.

A transmissão do Mycobacterium leprae está relacionada a fatores individuais (resposta imunológica) e sócio-econômicos, bem como, ao diagnóstico e tratamento. A convivência com doentes multibacilares propicia a transmissão do bacilo, especialmente em ambientes Que concentram elevado número de pessoas, em condições sócio-econômicas desfavoráveis ${ }^{(1)}$.

A evolução histórica da doença aponta para o estigma e o preconceito contra o doente, calcados na transmissibilidade e na possibilidade de mutilação do indivíduo. A investigação adeQuada dos contatos contribui para a interrupção da cadeia de transmissão da hanseníase, pois trata precocemente os casos diagnosticados, evitando a disseminação do bacilo e a instalação de incapacidades, pois estas podem limitar a produtividade do indivíduo e gerar a marginalização social.

No Brasil, como conseqüência das políticas adotadas pelo Ministério da Saúde, houve uma redução do número de casos, de 19 para, aproximadamente, cinco por 10.000 habitantes, no período de 1985 a 1999. Entretanto, ainda não se conseguiu atingir o índice aceito pela Organização Mundial da Saúde, ou seja, de menos de um caso para cada 10.000 habitantes $^{(1,2)}$.

O coeficiente de prevalência, em 2005, diminuiu para I,48 casos por 10.000 habitantes, equivalendo a 27.313 indivíduos em tratamento. A taxa de detecção foi de 2,09 por 10.000 habitantes, $\mathrm{o}$ Que corresponde à notificação de $38.410 \operatorname{casos}^{(3)}$. Observa-se, entretanto, Que o Brasil vem mantendo uma média de 47 mil casos novos por ano, no último Qüinqüênio ${ }^{(4)}$.

Em decorrência dos dados citados, o Programa Nacional de Controle da Hanseníase recomenda Que a doença seja inserida entre as prioridades do Pacto pela Saúde, fortalecendo as ações de vigilância da saúde desde a atenção básica à média e alta complexidade, salientando Que a maioria dos casos pode ser diagnosticada, tratada e curada na atenção básica ${ }^{(4)}$.

No Estado do Paraná, apesar do baixo coeficiente de prevalência em relação ao país ( 1,49 caso em 10.000 habitantes) ${ }^{(5)}$, o número de casos aumentou no período de 1994 a 2007, chegando a 1.865 casos novos em 2004. Embora tenha havido uma discreta diminuição, com 1.511 casos em 2006 ${ }^{(2)}$, o Paraná é o estado com maior prevalência na Região Sul do país.

Um estudo Que avalia a perspectiva de controle da hanseníase no Paraná indica Que o estado encontra-se próximo da meta da OMS. Entretanto, os autores salientam Que o coeficiente de detecção não diminuiu no decorrer dos seis anos do estudo, indicando importante circulação de bacilos e um número significativo de adoecimento por hanseníase no estado. Consideram, também, Que a redução no coeficiente de prevalência tenha ocorrido pelo aumento do número de altas e não pela diminuição de casos diagnosticados ${ }^{(6)}$.

No município de Londrina, o coeficiente de prevalência é de 1,37 e a taxa de detecção é de 1,15 por 10.000 habitantes, para a população geral, e de 0,08 , para menores de $15 \operatorname{anos}^{(5)}$. Esses índices indicam Que o município ainda não atingiu a meta de controle da hanseníase (taxa de prevalência menor de um caso por 10.000 habitantes). A taxa de detecção é considerada alta, segundo critérios do Ministério da Saúde ${ }^{(7)}$, o Que configura alta endemicidade e maior possibilidade de transmissão do bacilo.

Para alcançar a meta de controle da hanseníase da Organização Mundial da Saúde e pretendida pelo Ministério da Saúde até o ano 2010, torna-se necessário investir em ações efetivas para o diagnóstico precoce da doença ${ }^{(3)}$. O controle dos contatos intradomiciliares deve fazer parte dessas ações, pois os familiares são os indivíduos mais expostos à infecção.

O Ministério da Saúde ${ }^{(8)}$ indica Que as ações das unidades de saúde devem ser programadas, considerando-se uma média de Quatro contatos domiciliares por paciente. Este órgão define como contato intradomiciliar "toda e qualeuer pessoa Que resida ou tenha residido com o doente, nos últimos cinco anos”. Assim, para cada caso diagnosticado, deve-se prever a vigilância de Quatro indivíduos, com o objetivo de se adotar medidas profiláticas em relação aos mesmos. O diagnóstico precoce possibilita a adoção das medidas terapêuticas imediatas e adequadas a cada caso. Considera-se o controle dos comunicantes como um dos pilares para o controle da hanseníase ${ }^{(9)}$.

$\mathrm{O}$ atendimento da hanseníase, no município de Londrina-PR, tem encontrado dificuldades para a sua descentralização, devido aos muitos fatores envolvidos nessa ação. Embora as equipes de saúde das Unidades Básicas e da Saúde da Família tenham participado de treinamentos específicos, ainda não se observaram reflexos no diagnóstico precoce e nos coeficientes do município. O controle dos contatos parece estar distante de sua efetivação, o Que pode interferir no controle da endemia. A busca de casos e contatos constituía-se em problema para a saúde pública, mesmo Quando as ações eram centralizadas, período em Que foram levantados os dados deste estudo.

A falta de informações sobre a hanseníase e suas formas de transmissão coloca o próprio indivíduo como um ser passivo diante do controle da doença, pois muitos contatos domiciliares, a despeito da solicitação de comparecimento ao serviço de saúde para exame dermato-neurológico, acabam não comparecendo. Por outro lado, as eQuipes de saúde parecem não conseguir cumprir um programa de busca ativa desses contatos.

Diante de todas as dificuldades relacionadas, o presente estudo buscou responder ao seguinte Questionamento: como ocorreu a vigilância epidemiológica dos contatos intradomiciliares no período de 1996 a 2005 no município de Londrina?

Espera-se que os resultados encontrados nesta investigação, Que examina algumas variáveis relacionadas aos comunicantes, possam contribuir com as equipes e os serviços de saúde do município, para o controle da endemia.

O objetivo deste estudo é analisar algumas variáveis relacionadas aos contatos intradomiciliares de pacientes com hanseníase, atendidos no município de Londrina-PR, num período de dez anos.

\section{MÉTODO}

Estudo inter-relacional retrospectivo realizado junto às Fichas de Controle de Comunicantes, contidas nos prontuários e Fichas do Sistema Nacional de Agravos de Notificação (SINAN), dos pacientes atendidos com diagnóstico de hanseníase no serviço de referência 
do município de Londrina-PR.

Utilizou-se um instrumento pré-estruturado, contendo dados de identificação do contato (sexo, idade, grau de parentesco, região de moradia no município), assim como, informações sobre realização de avaliação dermato-neurológica, indicação e registro de vacinação BCG. O instrumento contém, ainda, dados do caso de hanseníase, como: forma clínica, resultado de baciloscopia e biópsia.

Este estudo é parte da pesquisa intitulada "Aspectos epidemiológicos da hanseníase no município de Londrina-PR nos últimos dez anos: possibilidade de controle da endemia?", cujo projeto foi aprovado pelo Comitê de Ética em Pesquisa da Universidade Estadual de Londrina (Parecer No 209/05 de 08/ I I/ 2005) e autorizado pela Direção do Centro Integrado de Doenças Infecciosas (CIDI), local Que centralizava os atendimentos dos pacientes e seus contatos. Os sujeitos da pesquisa tiveram sua identidade preservada, não sofrendo prejuízos decorrentes do estudo.

Os dados foram coletados no período de julho de 2006 a julho de 2007 e se referem aos contatos dos casos atendidos no período de janeiro de 1996 a dezembro de 2005, totalizando 1055 casos de hanseníase.

Os dados foram tabulados por meio do programa SPSS, e são apresentados de forma descritiva e em tabelas.

\section{RESULTADOS E DISCUSSÃO}

Analisando-se os prontuários dos 1.055 casos de hanseníase, atendidos no município de Londrina-PR, no período de 1996 a 2005 , observou-se que $883(83,7 \%)$ tinham contatos domiciliares registrados nas fichas de controle, totalizando 3.394 indivíduos. Ressalta-se Que, desse total, 32 pessoas $(0,9 \%)$, registradas como comunicantes, já eram também casos confirmados de hanseníase. A média foi de 3,2 contatos para cada caso, o Que se aproxima da média Quatro, recomendada pelo Ministério da Saúde ${ }^{(8)}$ para ser considerada no planejamento das ações de vigilância. A tabela 1 apresenta algumas características desses indivíduos.

Os indivíduos mais expostos ao $M$. leprae estão na faixa etária de até 40 anos de idade (71,5\%), sendo filhos/as, em 40,6\% dos casos e esposo/a ou companheiro/a, em 17,8\% (tabela 1). A exposição de adolescentes e jovens pode levar ao aparecimento de sinais da doença em idade produtiva, uma vez Que o período de incubação é longo. Há referências de nove meses a 20 anos $^{(10)}$, de dois a sete anos $^{(11)}$,e de 3 a 5 anos $^{(12)}$.

Observa-se Que todas as regiões do município de Londrina apresentam grande número de contatos, destacando-se as regiões Sul e Norte, além do elevado número de contatos de pacientes vindos de outro município $(22,9 \%)$. A procura pelos serviços de saúde de Londrina é uma constante, por este ser município de referência para várias especialidades da área de abrangência da $17^{\text {a }}$ Regional de Saúde do Paraná.

Dentre os 3394 contatos registrados na ficha de controle, apenas 1731 ( 51 1,0\%) passaram por avaliação dermato-neurológica. Destes, 183 (10,6\%) apresentavam algum sinal sugestivo de hanseníase. Foram descartados 47 casos e confirmados 16, o Que corresponde a $0,9 \%$ do total dos comunicantes examinados. Ressalta-se, entretanto, Que $120(6,9 \%)$ casos não concluíram a investigação, o Que pode contribuir para aumentar o número de casos ou retardar o diagnóstico. Salienta-se Que, além dos 183 contatos com sinais sugestivos de
Tabela 1. Distribuição dos contatos intradomiciliares de pacientes com hanseníase, segundo a faixa etária, o grau de parentesco e a região do município Que habitam. Londrina, 1996 a 2005. $(n=3.394)$.

\begin{tabular}{lcc}
\hline Faixa etária (anos) & $\mathbf{n}$ & \% \\
\hline Até I0 & 598 & 17,6 \\
11 a 20 & 806 & 23,7 \\
21 a 30 & 614 & 18,1 \\
31 a 40 & 411 & 12,1 \\
41 a 50 & 307 & 9,0 \\
51 a 60 & 223 & 6,6 \\
61 e mais & 233 & 6,9 \\
Ignorado & 202 & 6,0 \\
\hline Grau de parentesco & & \\
\hline Filho(a) & 1379 & 40,6 \\
Esposo(a)/companheir & 604 & 17,8 \\
o(a) & & \\
Irmão(ã) & 351 & 10,3 \\
Neto(a) & 252 & 7,4 \\
Pai/mãe & 251 & 7,4 \\
Sobrinho(a) & 116 & 3,4 \\
Avô(ó)/tio(a) & 40 & 1,2 \\
Outros & 268 & 7,9 \\
lgnorado/nada consta & 133 & 3,9 \\
\hline Região do município & & \\
\hline Norte & 477 & 14,1 \\
Sul & 740 & 21,8 \\
Leste & 279 & 8,2 \\
Oeste & 420 & 12,4 \\
Centro & 414 & 12,2 \\
Rural & 164 & 4,8 \\
Outro município & 776 & 22,9 \\
Ignorado & 124 & 3,7 \\
\hline
\end{tabular}

hanseníase, havia mais $32(0,9 \%)$ Que já eram casos confirmados.

A análise da situação do controle dos comunicantes na literatura mostra Que, a despeito dos grandes avanços na terapêutica da hanseníase, não se pode afirmar o mesmo em relação aos comunicantes $^{(13)}$. Os diferentes modelos de atenção à saúde contemplam ações de controle dos contatos, mas, na prática, estas não estão integradas às demais ações das unidades de saúde.

Considerando-se os dados apresentados, salienta-se a necessidade de: busca ativa dos contatos; acompanhamento dos mesmos; disponibilização de informações sobre a doença e os modos de transmissão para a população em geral.

A tabela 2 apresenta os dados sobre baciloscopia e forma clínica do caso. Os contatos domiciliares estão expostos a casos com baciloscopia, de lóbulos auriculares e cotovelos, positiva $(3 \mathrm{I}, 0 \%)$, e/ ou a casos em que houve o encontro do bacilo no exame anátomopatológico da lesão $(22,7 \%)$. Considerando-se que o homem é a única fonte de infecção do $M$. leprae, a exposição desses indivíduos, por período prolongado, aumenta a possibilidade de manutenção da endemia. Embora haja uma estimativa de Que $90,0 \%$ da população tenha resistência natural ao $M$. leprae, a convivência familiar é apontada como fator importante para a transmissão da doença ${ }^{(14)}$.

$\mathrm{O}$ contato com pacientes Que apresentam as formas dimorfa e virchowiana, cuja eliminação do bacilo é maior (formas multibacilares), 
correspondeu a 51 , 6\% dos contatos. Em grande parcela da população, não é possível estabelecer se houve exposição a casos conhecidos, mas a maioria dos estudos indica as formas multibacilares (virchowiana e dimorfa) como as de maior importância epidemiológica na transmissão da hanseníase ${ }^{(15)}$.

Os indivíduos paucibacilares não constituem fonte relevante de transmissão do $M$. leprae, pela baixa carga bacilar ${ }^{(11)}$.

As taxas de incidência da hanseníase entre contatos domiciliares são, pelo menos 12 vezes maiores, em casos predominantemente multibacilares $^{(16)}$.

Avaliando-se alguns estudos sobre o risco para os contatos de hanseníase, a maioria demonstra índices de euatro a dez vezes maiores do Que os apresentados para a população em geral, Quando se trata de exposição a casos multibacilares ${ }^{(9)}$.

Os dados reforçam a necessidade do diagnóstico precoce e da

Tabela 2.Distribuição dos contatos intradomi-ciliares de pacientes com hanseníase, segundo resultado da baciloscopia e forma clínica do caso. Londrina, 1996 a 2005. $(n=3.394)$.

\begin{tabular}{lcc}
\hline $\begin{array}{l}\text { Baciloscopia } \\
\text { (lóbulos auriculares e cotovelos) }\end{array}$ & $\mathbf{n}$ & $\%$ \\
\hline Positiva & 1054 & 31,0 \\
Negativa & 1648 & 48,6 \\
Não realizada/ignorada & 692 & 20,4 \\
\hline Baciloscopia & & \\
(biópsia da lesão) & & \\
\hline Positiva & 770 & 22,7 \\
Negativa & 1099 & 32,4 \\
Não realizada/ignorada & 1525 & 44,9 \\
\hline Formas Clínicas & & \\
\hline Indeterminada & 582 & 17,1 \\
Tuberculóide & 1029 & 30,3 \\
Dimorfa & 779 & 23,0 \\
Virchowiana & 970 & 28,6 \\
Não classificada & 34 & 1,0 \\
\hline
\end{tabular}

Tabela 3. Distribuição dos contatos intradomiciliares de pacientes com hanseníase, segundo avaliação e indicação da vacina BCG. Londrina, 1996 a 2005. $(n=3.394)$.

\begin{tabular}{ccc}
\hline Indicação BCG & $\mathbf{n}$ & \% \\
\hline Sim, uma dose & 757 & 22,3 \\
Sim, duas doses & 344 & 10,1 \\
Não indicado & 129 & 3,8 \\
Nada consta & 573 & 16,9 \\
Não avaliado* & 1591 & 46,9 \\
\hline I dose recebida $^{\text {Sim }}$ & \\
\hline Não & 664 & 19,6 \\
Ignorado* & 20 & 0,6 \\
\hline $2^{\text {a dose recebida }}$ & 2710 & 79,8 \\
\hline Sim & 141 & 4,2 \\
Não & 29 & 0,9 \\
Ignorado* & 3224 & 95,0 \\
\hline *Incluídos os contatos Que não compareceram para avaliação.
\end{tabular}

instituição de tratamento adequado, o Que contribui para diminuir o período de exposição dos contatos aos casos, principalmente, aos multibacilares.

O estado vacinal, em relação à BCG, faz parte das recomendações do Ministério da Saúde para o controle dos contatos. A tabela 3 mostra a indicação e a efetivação da vacina BCG, conforme anotações encontradas nas fichas dos comunicantes.

Ressalta-se Que 46,9\% dos contatos não foram avaliados em relação ao estado vacinal, mesmo porQue não passaram por avaliação clínica. Em 16,9\%, não havia registro dessa indicação. Esses dados são reflexos da ausência dos contatos ao serviço, para a avaliação dermato-neurológica, assim como, para a avaliação de indicação da vacina $B C G$.

A efetivação da primeira dose de BCG foi considerada pela presença de cicatriz vacinal, no momento da avaliação clínica, ou Quando havia anotação da data de realização da mesma. O mesmo critério foi adotado para a segunda dose. Salienta-se o grande número de contatos em Que não há informação sobre a administração da primeira $(79,8 \%)$ e da segunda dose $(95,0 \%)$. Entretanto, não se pode afirmar Que esses indivíduos não receberam a vacina BCG, mas fica evidente a falta de controle do serviço sobre estes dados.

A eficácia da vacina BCG, na imunoprofilaxia e na imunoterapia da hanseníase, tem sido tema de vários estudos, observando-se uma variação muito grande nesse índice. A análise de três estudos realizados na Uganda, em Nova Guiné e na Birmânia mostra uma variação de 20 a 80\% na eficácia da BCG para a profilaxia da hanseníase, justificada, provavelmente, pelas diferenças metodológicas adotadas em cada estudo $^{(17)}$. Apesar de apresentarem resultados contraditórios, a maioria das pesquisas aponta benefícios tanto na profilaxia como na terapêutica associada à polieuimioterapia ${ }^{(18)}$. Tanto a BCG oral como a intradérmica produzem viragem imunológica do Mitsuda, de negativo para positivo.

No Brasil, o número de doses da vacina BCG tem variado no decorrer dos anos e em diferentes estados. O Ministério da Saúde, Que recomendava duas doses com intervalo de seis meses ${ }^{(1)}$, passa a adotar nova conduta, não indicando o aprazamento do contato para a segunda dose. Assim, em contato considerado indene (não-doente), deve-se avaliar a cicatriz vacinal de BCG e seguir a seguinte orientação: uma dose, na ausência ou na presença de uma cicatriz; não vacinar no caso de apresentar duas cicatrizes ${ }^{(14)}$.

O Ministério da Saúde ainda recomenda atenção especial na investigação de contatos de pacientes menores de 15 anos, o Que se justifica pela possibilidade de transmissão recente e ativa ${ }^{(14)}$.

Avaliando-se os dados apresentados neste estudo, constata-se a fragilidade dos serviços de saúde do município de Londrina para o controle dos contatos, tanto na busca ativa para avaliação dermatoneurológica, Quanto no acompanhamento desses indivíduos para novas avaliações e efetivação da vacina BCG.

\section{CONCLUSÃO}

A análise dos 3394 contatos, registrados nos prontuários dos 1055 casos de hanseníase atendidos no município de Londrina, mostra Que a maioria é composta por uma população jovem, sendo filhos/as e esposos/as os parentes mais expostos. As regiões Sul e Norte apresentam mais contatos, embora os de outros municípios também sejam relevantes.

Dentre os contatos registrados, apenas metade $(51,0 \%)$ passou 
por avaliação dermato-neurológica, sendo Que 183 apresentavam algum sinal sugestivo de hanseníase, dos Quais 120 não concluíram a investigação.

A análise das informações relativas à positividade da baciloscopia e das formas clínicas multibacilares permite afirmar que grande parte dos contatos estão expostos a cargas bacilares Que os colocam em risco de adQuirir a infecção.

A avaliação dos contatos a respeito da indicação e efetivação da vacina BCG sugere que o acompanhamento e o controle desses indivíduos, pelo serviço, não estão sendo efetivos.

Os dados reforçam a necessidade de ações Que proporcionem: o diagnóstico precoce, especialmente entre os contatos intradomiciliares; a instituição de tratamento adeQuado; o acesso às informações sobre a doença e os modos de transmissão, diminuindo assim, o período de exposição dos contatos aos casos multibacilares.

A descentralização das ações coloca as equipes das unidades básicas de saúde e de saúde da família como principais pilares para a melhoria da Qualidade da assistência e o real controle da endemia.

\section{REFERÊNCIAS}

1. Ministério da Saúde (BR). Hanseníase: atividades de controle e manual de procedimentos. Área Técnica de Dermatologia Sanitária. Brasília: Ministério da Saúde; 2001 .

2. Machado K. Controle da hanseníase: agora de olho nos jovens. Radis 2008; 68: 10-3.

3. Ministério da Saúde (BR). Secretaria de Gestão Estratégica e Participativa. Departamento de Monitoramento e Avaliação da Gestão do SUS. Painel de indicadores do SUS. Brasília: Ministério da Saúde; 2006.

4. Ministério da Saúde (BR). Secretaria de Atenção à Saúde. Departamento de Atenção Básica. A responsabilidade da atenção básica no diagnóstico da hanseníase. Informe da atenção básica; Brasília: Ministério da Saúde; 2007.

5. Ministério da Saúde (BR). SESA/SINAN. Hanseníase - dados do Paraná; 2005. [citado em: 19 ago 2008]. Disponível em: URL: http://www.saude.pr.gov.br/

6. Silva Sobrinho RA, Mathias TAF. Perspectivas de eliminação da hanseníase como problema de saúde pública no Estado do Paraná, Brasil. Cad Saúde Pública 2008; 24(2): 303-I 4.

7. Ministério da Saúde (BR). Secretaria de Vigilância em saúde. Departamento de Vigilância Epidemiológica. Programa Nacional de Controle da Hanseníase. Indicadores de monitoramento do comportamento da hanseníase no Brasil: Nota técnica N No. $010 /$ 2007/PNCH/DEVEP/SVS/MS. Brasília: Ministério da Saáude; 2007. [citado em: 17 jul 2008]. Disponível em: http:// portal.saude.gov.br/portal/arQuivos/pdf/indicadores hansen.pdf

8. Ministério da Saúde (BR). Secretaria de Políticas de Saúde. Departamento de Atenção Básica. Guia para o controle da hanseníase. Brasília: Ministério da Saúde; 2002.
9. Pinto Neto IM, Villa TCS, Mencaroni DA, Gonzáles RC, Gazeta CE. Considerações epidemiológicas referentes ao controle dos comunicantes de hanseníase. Hansen Int 2002; 27( I): 23-28.

10. Chin I. Manual de controle das doenças transmissíveis. $17^{\text {a }}$ ed. Porto Alegre: Artmed; 2002.

11. Ministério da Saúde (BR). Secretaria de Vigilância em Saúde. Guia de vigilância epidemiológica. Brasília: Ministério da Saúde; 2005.

12. Margarido LC, Rivitti EA. Hanseníase. In: Veronesi R, Focacia R. Tratado de infectologia. São Paulo: Atheneu; 2006. p. 937-69.

13. Pinto Neto JM, Villa TCS, Oliveira MHP, Barbeira CBS. O controle dos comunicantes de hanseníase no Brasil: uma revisão da literatura. Hansen Int 2000; 25(2): I63-76.

14. Ministério da Saúde (BR). Secretaria de Atenção à Saúde. Departamento de Atenção Básica. Vigilância em saúde: dengue, esquistossomose, hanseníase, malária, tracoma e tuberculose. Cad Atenção Vásica 2007; 21 .

15. Néri JAC, Sales AM, Luz M. Vigilância epidemiológica da hanseníase. In: Aguiar AC, Reis ACGV, Sales AM, Fonseca AF, Rodrigues DP, Carvalho DM, et al. Textos de apoio em vigilância epidemiológica. Rio de Janeiro: Fiocruz; 1998. p. 7 I-8.

16. Matos HI, Duppre N, Alvim MFS, Vieira LMM, Sarno EM, Struchtner. Epidemiologia da hanseníase em coorte de contatos intradomiciliares no Rio de Janeiro (1987-1991). Cad Saúde Pública 1999; 15(3): 533-42.

17. Pazin Filho A, Bueno CDF, Pinheiro SA, Ruffino Netto A. Estudos de intervenção sobre vacinação BCG em hanseníase: uma análise comparativa. Med Ribeirão Preto 1993; 26(3): 387-92.

18. Azulay RD. Vacinação anti-hansênica. Anais Bras Dermatol 2002; 77(4): 489-94. 